\title{
Cyclic variations in demand for out-of-hours services in child and adolescent psychiatry: implications for service planning
}

Thomas Hillen, ${ }^{1}$ Eduardo Szaniecki ${ }^{2}$

The Psychiatrist (2010), 34, 427-432, doi: 10.1192/pb.bp.109.026740

${ }^{1}$ Tavistock and Portman NHS Foundation Trust: ${ }^{2}$ Peace Children's Centre, Hertfordshire Partnership Foundation Trust

Correspondence to Thomas Hillen (thillen2@tavi-port.nhs.uk)

\begin{abstract}
Aims and method We examined peaks and troughs in demand for out-of-hours consultations and assessments in child and adolescent psychiatry and whether these have implications for service development. All out-of-hours referrals in three teaching hospitals providing services to a large and diverse inner-city population were prospectively registered from 1 January 2002 to 31 December 2005.
\end{abstract}

Results In total, 323 individuals under 18 years were referred; $21.6(95 \% \mathrm{Cl} 19.3-$ 24.0) per 100000 general population per year. Bedside assessments were required in $37 \%$, the remainder were dealt with by telephone. The work took more than $4 \mathrm{~h}$ for $22 \% ; 46 \%$ of individuals presenting were aged 16-18 years. Significant diurnal variation was observed with a peak in referrals between $17.00 \mathrm{~h}$ and $00.59 \mathrm{~h}$. More referrals were made when daytime liaison services were closed (odds ratio $(O R)=2.46,95 \% \mathrm{Cl} 1.15-1.87)$, after referral pathways had been clarified $(O R=2.33$, $95 \% \mathrm{Cl} 1.92-3.23)$ and during spring $(\mathrm{OR}=1.50,95 \% \mathrm{Cl} 1.14-1.98)$. Other factors analysed showed no association with referral activity.

Clinical implications Cyclic variations in the demand for out-of-hours services in child and adolescent psychiatry should be considered for service planning. Although paediatric psychiatric emergencies are relatively rare, they can take up considerable clinician time.

Declaration of interest None.
Paediatric psychiatric emergencies typically comprise self-harm, acute manifestations of mental illness and severe behavioural disturbances including violence. ${ }^{1}$ Frequently, young people in psychosocial crisis situations also present to hospital. Accident and emergency (A\&E) departments have aptly been described as 'the only game in town' outside normal working hours. ${ }^{2}$ This is where young people in a crisis situation take themselves or are taken by others, and their numbers are increasing even though professional help is not always sought. ${ }^{3,4}$ Often help is initially offered by paediatric nurses and doctors, who then liaise with child and adolescent psychiatrists. ${ }^{5}$

Guidance regarding the management of paediatric psychiatric emergencies has mainly been published with regard to self-harm; and the need for a comprehensive specialist psychosocial assessment has been emphasised in the UK since $1998{ }^{6,7}$ Although improved and timely access to specialist services is a UK government target, currently only a quarter of child and adolescent mental health services (CAMHS) provide emergency cover outside normal working hours. ${ }^{8,9}$ Many CAMHS still have to establish emergency duty services. A good understanding of when demand is likely to be at its greatest will help with service planning. So far, research into cyclic variations in psychiatric emergencies has mainly focused on adults and self-harm. How often and when individuals present to psychiatric emergency services is influenced by the organisation of the wider support networks, referral pathways and admission policies. Considerable variation in these service-dependent factors has been reported. ${ }^{10-12}$ However, fluctuations in emergency presentations have also been linked to service-independent factors such as diurnal, seasonal and meteorological variations. For example, more individuals present in the evening and at weekends, in spring, and on days with nice weather. ${ }^{13-16}$

This study set out to investigate cyclic variations in demand for paediatric psychiatric provision outside normal working hours in a large and diverse urban population. We wanted to find out whether service-dependent factors such as the availability of liaison daytime cover, the introduction of $\mathrm{A} \& \mathrm{E}$ waiting targets or high $\mathrm{A} \& \mathrm{E}$ workload were associated with demand for out-of-hours provision. We also investigated whether service-independent factors such as school holidays, the season and the weather related to demand. 


\section{Method}

\section{Setting}

This study was based at three inner-city teaching National Health Service (NHS) hospitals in north London and included University College London Hospitals, the Royal Free Hospital and the Whittington Hospital. All three sites had A\&E departments providing year-round 24-hour access and paediatric in-patient facilities. The hospitals provided services for young people presenting in crisis situations. All three hospitals worked closely with the local CAMHS, which were open on working days between $09.00 \mathrm{~h}$ and $17.00 \mathrm{~h}$. The three hospitals catered mainly for the boroughs of Camden and Islington, with a combined population of 373817 and high ethnic and socioeconomic diversity according to 2001 census data. ${ }^{17}$ However, the hospitals did not have clearly defined catchment areas and young people frequently came from neighbouring boroughs or other regions of the UK owing to the hospitals' proximity to major railway links.

The out-of-hours service in child and adolescent psychiatry was provided by senior trainee doctors undertaking specialist training at the Tavistock Clinic (specialist registrars), who had access to consultant cover at all times. The trainee doctors consulted with the paediatricians and junior trainees in general psychiatry (senior house officers) at the three hospital sites. Young people in crisis were usually first dealt with by professionals on site, who then decided whether or not input from the out-of-hours service in child and adolescent psychiatry was required.

The vast majority of young people accessing the out-ofhours service did so by presenting to A\&E. A minority were already in-patients on paediatric wards having been admitted for physical illness or psychosocial reasons and their paediatricians asked for management advice. Young people could self-present to $\mathrm{A} \& \mathrm{E}$, or they were brought to A\&E by their parents, their social worker or the police. General practitioners (GPs) could also organise a transfer to A\&E. Yong people referred to the out-of-hours service typically presented with self-laceration or overdoses compounded by uncooperative behaviour or severe family dysfunction, acute episodes of mental illness or psychosocial crises leading to a need for an urgent placement outside the family home. These presentations often generated high levels of concern in the professional network.

\section{Data collection}

Data on all referrals to the out-of-hours service in child and adolescent psychiatry were collected prospectively. Trainee doctors were required to complete an electronic audit form for each referral and forms were collected centrally. Regular reminders were sent out to ensure complete ascertainment of all referrals. Monthly on-call supervision meetings were held to maximise consistency between trainees. Data collection was fully compliant with the Data Protection Act of 1998. The current study solely relied on data routinely collected for service evaluation and did not require ethics review by the NHS National Research Ethics Service.

The date and time of the referral was recorded, as was the individual's date of birth and gender. The on-call doctor documented the reason for the referral, how long they spent dealing with it, whether it was a bedside assessment or telephone consultation, and whether or not the referral was deemed appropriate for the out-of-hours service in child and adolescent psychiatry. The present study includes all young people aged 18 and under referred to the out-of-hours service in child and adolescent psychiatry in the period between 1 January 2002 and 31 December 2005.

\section{Variables analysed}

The daily referral rate was the dependent variable and the independent variables investigated are listed below.

\section{No daytime cover}

A child and adolescent psychiatry hospital liaison service was available during normal working days at each of the three hospital sites. The referral frequency on days with daytime liaison cover and those without (e.g. weekends, public holidays) was compared.

\section{A\&E workload}

The number of people waiting for 3 or more hours on each day was obtained for the three hospital sites. The A\&E workload was defined as 'high' on days when the number of individuals waiting fell into the upper quartile for the site. The site-specific quartiles were calculated separately for each year.

\section{Changeover of senior house officers}

At each of the three hospitals, all junior doctors working in A\&E changed jobs in the same week in February and August. The referral frequency during the 8 weeks before changeover was compared with that of the 4 weeks afterwards.

\section{Protocol changes}

All three sites used different protocols to detail the referral pathways to the out-of-hours service in child and adolescent psychiatry. These were amended with the aim of tightening referral procedures and increasing the number of appropriate referrals at all three sites at different time points. In order to analyse whether the protocol changes resulted in a change in the referral frequency, the last 100 days before the change were compared with the first 100 days afterwards for each site.

\section{Introduction of the 4-hour A\&E waiting time target}

Hospitals in England were paid incentives when they met 4-hour A\&E waiting time targets as of 1 January 2004. The referral rates in the years before and after the introduction of the target were compared.

\section{School holidays}

The school dates of the borough of Camden were used to compare the referral frequency on days when pupils were expected to be in school with days when they were not.

\section{Spring}

The referral frequency for days in spring (21 March to 21 June) was compared with that of the other days in the year. 


\section{Hot and rainy days}

Meteorological data were obtained from the Meteorological Office for each day during the study period. Days with a mean temperature above $20^{\circ} \mathrm{C}$ (i.e. $9.1 \%$ of all days during the study period) were coded as 'hot' and those with more than $4 \mathrm{~mm}$ rainfall as 'rainy' (i.e. $11.2 \%$ ).

\section{Statistical analysis}

The chi-squared test was used to compare the distribution of referrals in the specified period with its comparison period (e.g. normal working days $v$. holidays). Odds ratios and their confidence intervals were calculated using EpiInfo6 (Centers for Disease Control and Prevention, USA; www.cdc.gov/epiinfo/epi6/ei6.htm) on Windows XP Professional. Stratification according to hospital site was used for the analysis of service-dependent variables and pooled odds and their confidence intervals were obtained by using the Mantel-Haenszel method. Whether pooled odds ratios could be used or whether differences over strata were significant was assessed by the Breslow-Day test. Multivariate analyses were not performed because of the covariation of the different independent variables under investigation. $^{18}$

For the power calculation it was assumed there would be one referral every 4 days and that $20 \%$ of the days in the 4-year study period carried an increased risk of a referral (e.g. no daytime cover). Given this, the present study had $81.5 \%$ power to detect odds ratios of 1.5 or greater with $\alpha=0.05$.

\section{Results}

Between I January 2002 and 31 December 2005 a total of 323 young people were referred to the out-of-hours service in child and adolescent psychiatry. Of these, 118 (36.5\%) required a bedside assessment, whereas for $205(63.5 \%)$ a consultation was given on the telephone. Based on 2001 census data, ${ }^{17}$ this equals to 21.6 (95\% CI 19.3-24.0) referrals per 100000 general population per year for either telephone consultations and bedside assessments taken together and 7.9 (95\% CI 6.5-9.3) per 100000 general population per year for bedside assessments only. There was no significant fluctuation in the yearly referral rate over the 4-year period, which was 76 in 2002 (95\% CI 59-93), 89 in 2003 and 2004 (95\% CI 71-107) and 69 in 2005 (95\% CI 53-85) $\left(\chi^{2}=3.024\right.$, d.f. $\left.=3, P=0.39\right)$.

Details of the referrals received are given separately for bedside assessments and for telephone consultation and bedside assessment combined in Table 1. The breakdown according to age revealed almost half of the referrals concerned young people over 16 years of age.

The diurnal distribution of referrals is shown in Fig. 1. Throughout the day the peak in total referrals occurred between $17.00 \mathrm{~h}$ and $00.59 \mathrm{~h}$, and the trough in the referral activity was between $01.00 \mathrm{~h}$ and $08.59 \mathrm{~h}$. There was an additional peak in referral activity between $09.00 \mathrm{~h}$ and $12.59 \mathrm{~h}$, which was as a result of referrals received on days without daytime liaison cover.

Of the 205 referrals leading to a telephone consultation, $167(81.5 \%)$ could be dealt with in less than $1 \mathrm{~h}$. However, when trainee doctors had to go into the hospital to undertake a bedside assessment $(n=118)$, they spent more than $4 \mathrm{~h}$ on dealing with the referral for 60 (50.5\%) individuals and for $16(13.6 \%)$ it took more than $6 \mathrm{~h}$.

The proportion of referrals considered appropriate by the trainee doctors was $79.0 \%$ and when only bedside assessments were considered this proportion rose to $89.8 \%$. After changes were made to the referral protocol there was an increase in appropriate and decrease in inappropriate referrals at all three sites. Accordingly, when the proportion of appropriate referrals before and after the protocol changes were compared, a pooled odds ratio of 3.9 (95\% CI 2.2-7.1) was obtained.

Table 2 provides information about how variables dependent on organisation of the service and those

\begin{tabular}{|c|c|c|}
\hline & \multicolumn{2}{|l|}{$n(\%)$} \\
\hline & $\begin{array}{l}\text { Phone consultation and bedside assessment combined } \\
\qquad(n=323)\end{array}$ & $\begin{array}{l}\text { Bedside assessment only } \\
\qquad(n=118)\end{array}$ \\
\hline Gender, male & $110(34)$ & $48(41)$ \\
\hline $\begin{array}{c}\text { Age, years } \\
0-12 \\
13-15 \\
16-18\end{array}$ & $\begin{array}{r}36(11.0) \\
139(42.9) \\
148(46.0)\end{array}$ & $\begin{array}{l}13(11.0) \\
51(43.5) \\
54(45.2)\end{array}$ \\
\hline $\begin{array}{c}\text { Referral time } \\
09.00-12.59 \\
13.00-16.59 \\
17.00-20.59 \\
21.00-00.59 \\
01.00-04.59 \\
05.00-08.59\end{array}$ & $\begin{array}{l}55(17.0) \\
34(10.5) \\
93(28.8) \\
86(26.6) \\
41(12.7) \\
14(4.3)\end{array}$ & $\begin{array}{c}34(28.8) \\
18(15.3) \\
28(23.7) \\
27(22.9) \\
7(5.9) \\
4(3.4)\end{array}$ \\
\hline $\begin{array}{c}\text { Time taken } \\
0-1 \mathrm{~h} \\
1-4 \mathrm{~h} \\
>4 \mathrm{~h}\end{array}$ & $\begin{array}{r}170(52.6) \\
81(25.2) \\
72(22.2)\end{array}$ & $\begin{array}{c}3(2.8) \\
55(46.7) \\
60(50.5)\end{array}$ \\
\hline Referral was appropriate & $255(79.0)$ & $106(89.8)$ \\
\hline
\end{tabular}




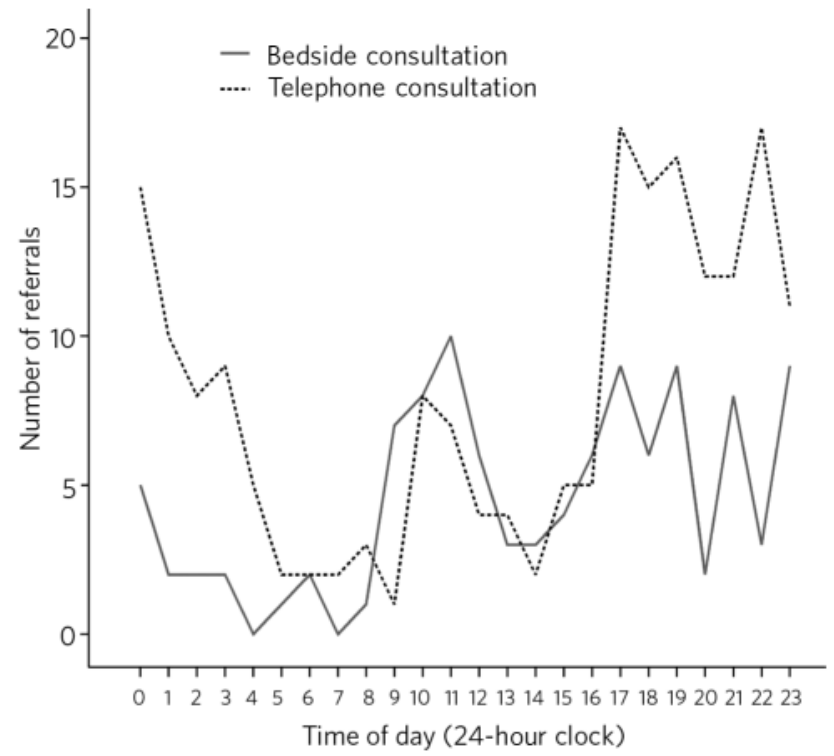

Fig 1 Diurnal variation in referrals to out-of-hours services in child and adolescent psychiatry.

independent from it related to the referral rate. The presence or absence of daytime liaison cover and protocol changes showed a significant association with both the referral rate for bedside consultation and telephone and bedside consultation combined. The proportion of referrals leading to a bedside assessment was significantly higher on days without daytime cover $(48 \%)$ than on those with cover (29\%, $P=0.001$ ). Data pertaining to service-dependent factors are given as pooled odds ratios since there were no significant differences over strata. Of the service-independent variables, only season reached significance and there were more referrals in spring than the rest of the year.

\section{Discussion}

The present study set out to identify peaks and troughs in the workload of out-of-hours services in child and adolescent psychiatry covering three inner-city teaching hospitals. Our data can help with service-planning and lead to a better understanding of the precipitants of paediatric psychiatric emergencies. The results show such out-of-hours services should expect around 22 referrals per 100000 population per year. Demand for provision outside normal working hours was at its greatest over weekends and between $17.00 \mathrm{~h}$ and $00.59 \mathrm{~h}$ on weekdays, which is in keeping with other studies focusing mainly on adults. ${ }^{14,15}$ In the majority of cases a telephone consultation to the attending paediatrician or psychiatric senior house officer was sufficient to draw up a management plan until the young person could be assessed by the regular daytime liaison team. On days without normal daytime cover, young people still required specialist mental health assessments and hence the peak in referrals between $09.00 \mathrm{~h}$ and $12.59 \mathrm{~h}$ and the higher proportion of bedside assessments on those days. In a significant proportion of cases (23\%) the bedside assessment could not be delayed until the next morning and the on-call child and adolescent psychiatrist had to see the young person between $17.00 \mathrm{~h}$ and $09.00 \mathrm{~h}$.

Nearly half of all referrals concerned young people aged 16 and over. This means that widening access to child and adolescent mental health services to include this age group, as required in England, is associated with a considerable increase in workload with resource implications. ${ }^{8}$ Many paediatric wards with a high proportion of babies and very young children are not an adequate environment for older adolescents. Paediatricians and mental health professionals not used to dealing with older adolescents might need additional training and support when they are expected to provide services to this group. The present study also revealed paediatric psychiatric emergencies can be time consuming, with over half of bedside assessments taking over $4 \mathrm{~h}$. Part of the reason for this lies in the complex nature of the work and difficulties with gathering relevant information after normal working hours. ${ }^{6}$ However, difficulties over arranging in-patient admission were, as elsewhere in the UK, one of the most frequent causes for delay in the catchment area studied. ${ }^{19}$

Previous research has demonstrated how service design and planning can influence how effectively out-of-hours

\begin{tabular}{|llr|}
\hline Table 2 & $\begin{array}{l}\text { Odds ratios for receiving referrals according to whether or not the examined service-dependent or service- } \\
\text { independent factors were present }\end{array}$ & \multicolumn{1}{c|}{ Odds ratio $(95 \% \mathrm{Cl})$} \\
\cline { 2 - 3 } & Bedside assessment and telephone consultation combined & Bedside assessment only \\
\hline Service-dependent factors ${ }^{\mathrm{a}}$ & & \\
No daytime cover & $1.46(1.15-1.87)$ & $2.32(1.60-3.43)$ \\
High A\&E workload & $1.05(0.72-1.53$ & $1.30(0.82-2.06)$ \\
4-hour A\&E time target introduced & $1.00(0.69-1.44)$ & $0.83(0.56-1.21)$ \\
Senior house officer changeover & $1.14(0.78-1.65)$ & $1.47(0.76-2.87)$ \\
Protocol changes & $2.33(1.92-3.23)$ & $2.08(1.41-3.23)$ \\
\hline Service-independent factors & & $1.50(0.75-1.43)$ \\
School holidays & $0.97(0.72-1.30)$ & $2.03(1.34-3.09)$ \\
Spring & $1.50(1.14-1.98)$ & $0.92(0.44-1.86)$ \\
Hot day $>20^{\circ} \mathrm{C}$ & $1.47(0.97-2.23)$ & $0.89(0.46-1.71)$ \\
Rainy day $>4 \mathrm{~mm}$ & $1.22(0.82-1.80)$ & \\
\hline
\end{tabular}

$A \& E$, accident and emergency.

a. For service-dependent factors stratification by hospital site was used and pooled odds ratios are given. 
services in child psychiatry are used. ${ }^{20}$ The present study showed an overall increase in referrals and simultaneous decrease in inappropriate referrals after clarification of referral pathways and criteria. Specifying that referrals should come from paediatricians or trainee psychiatrists proved a particularly effective measure. It should be acknowledged that the rating of the appropriateness of referrals was based on the judgement of the recipient of the referral. Other service-dependent factors examined in this study were not associated with how the out-of-hours service was used and how many referrals it received. For example, we had hypothesised that there would be more referrals when A\&E had a high workload, when the NHS 4-hour waiting time target was introduced or junior doctors rotated jobs. However, none of these factors showed a significant association with the referral rate.

Large epidemiological studies have shown cyclic variations in psychiatric emergencies depending on season, weather or holidays. ${ }^{13,15,16,21}$ This study analysed whether these factors would also be reflected in the referral pattern to out-of-hours service in child and adolescent psychiatry. The results showed no more referrals than usual during the holidays or when it was hot or rainy. However, there were $50 \%$ more referrals during spring than the rest of the year. This by far exceeds the seasonal fluctuations observed in other studies. One possible explanation is that many students in the UK sit important exams or experience other disruptions to their school life during spring (for example, 16-year-olds are on their last term of compulsory education). School stress has previously been linked to selfharm. ${ }^{22}$ However, the spring excess in referrals was not confined to those age groups most affected by the school situation. If the relatively large excess in referrals of young people in crisis during spring is replicated in further studies, it would be important to understand the reasons behind it better and to take this into account for service planning.

The methodological strengths of this study include the prospective data collection, the inclusion of three different sites/hospitals, the large sample size and diversity of the underlying population and the long period of observation. One of the limitations of the study was the use of only one source, the trainee doctors, for case ascertainment. When trainee doctors failed to record a referral when on call, the case was missed. The three hospitals did not have very well-defined catchment areas and this may have distorted the estimate of demand per 100000 general population. Generalisations from our data to other services might be difficult since the study was solely based on an inner-city population and services in other parts of the country may have a different configuration. ${ }^{10,12}$ Our study only recorded the day and time when the out-of-hours service received the referral and there was no information as to when the young person arrived at $\mathrm{A} \& \mathrm{E}$ or when the crisis (e.g. taking an overdose) started. This made comparison with some studies, which had this information, more difficult. Furthermore, without multivariate analyses there was no ability to control for confounding factors. Finally, children and young people in need of mental health assessment come to hospital through a variety of pathways such as self-referral and professional referrals by GPs, social workers and school nurses. In the current study the focus was on individuals that had found their way to the hospital and were then referred to the out-of-hours service in child and adolescent psychiatry. Further study into primary referral sources might advance our understanding of pathways, with possible consequences for service configuration and allocation of resources.

This study suggests that although paediatric psychiatric emergencies are relatively rare they can take up considerable clinician time and require input from professionals experienced in this field. Bundling services together to cover large catchment areas could be one way of ensuring that resources for maintaining out-of-hours provision are well used and that the clinicians involved see enough cases to develop the relevant expertise.

\section{Acknowledgements}

We particularly want to thank Dr Louise Richards for setting the case registry up when she was a trainee on the Tavistock Rotation. All trainee doctors participating in the on-call rota helped with the collation of the data. Drs S. Lewis, Z. Davis, M. Berelowitz and S. Kraemer reliably offered clinical supervision and kindly helped with editing the manuscript. We are grateful to the Met Office for providing us with the weather data.

\section{About the authors}

Dr Thomas Hillen is a consultant child and adolescent psychiatrist at the Tavistock and Portman NHS Foundation Trust, London. Dr Eduardo Szaniecki is a consultant child and adolescent psychiatrist at the Watford Child and Family Clinic, Peace Children's Centre, Hertfordshire Partnership Foundation Trust.

\section{References}

1 Edelsohen G, Braitman LE, Rabinovisch $H$, Sheves P, Melendez A. Predictors of urgency in a pediatric psychiatric emergency service. J Am Acad Child Adolesc Psychiatry 2003; 42: 1197-202.

2 Soskis CW. Emergency room on weekends: the only game in town. Health Social Work 1980; 5: 37-43.

3 Grupp-Phelan J, Harmon J, Kelleher KJ. Trends in mental health and chronic condition visits by children presenting for care at US emergency departments. Public Health Rep 2007; 122: 55-61.

4 Mental Health Foundation. Truth Hurts. Report of the National Inquiry into Self-harm among Young People. Mental Health Foundation, 2006.

5 Street C. Whose Crisis? Meeting The Needs of Children and Young People with Serious Mental Health Problems. Young Minds, 2000.

6 National Institute for Health and Clinical Excellence. Self-Harm: The Short-Term Physical and Psychological Management and Secondary Prevention of Self-Harm in Primary and Secondary Care. The British Psychological Society and The Royal College of Psychiatrists, 2004.

7 Royal College of Psychiatrists. Managing Deliberate Self-Harm in Young People. Council Report CR64. Royal College of Psychiatrists, 1998.

8 HM Government. Every Child Matters: Change for Children. Department for Education and Skills, 2004

9 Storey P, Statham J. Emergency support: whose crisis now? Community Care 2007; 11 October (http://www.communitycare.co.uk/Articles/ 2007/10/11/106086/mental-health-crisis-among-young-peopleresearch.htm)

10 Bennewith O, Gunnell D, Peteres TJ, Hawton K, House A. Variation in the hospital management of self-harm in adults in England: observational study. BMJ 2004; 328: 1108-9.

11 Hawton K, Bergen H, Cassey D, Simkin S, Plamer B, Cooper J, et al. Selfharm in England: a tale of three cities. Soc Psychiatry Psychiatr Epidemiol 2007; 42: 513-21. 
12 Rushton J, Bruckman D, Kelleher K. Primary care referral of children with psychosocial problems. Arch Pediatr Adolesc Med 2002; 156: 592-8.

13 Bergen $\mathrm{H}$, Hawton K. Variation in deliberate self-harm around Christmas and New Year. Soc Sci Med 2007; 65: 855-67.

14 Bergen $\mathrm{H}$, Hawton $\mathrm{K}$. Variations in time of hospital presentation for deliberate self-harm and their implications for clinical services. J Affect Disord 2007; 98: 227-37.

15 Blenkiron P. The timing of deliberate self harm behaviour. Ir J Psychol Med 2003; 20: 126-31.

16 Jessen G, Steffensen P, Jensen BF. Seasons and meteorological factors in suicidal behaviour. Arch Suicide Res 1998; 4: 263-80.

17 Office for National Statistics. Census 2001: the most comprehensive survey of the UK population. ONS, 2001 (http:/www.statistics.gov.uk/ census2001/census2001.asp).
18 Hakko H, Rasanen P, Tiihonen J, Nieminen P. Use of statistical techniques in studies of suicide seasonality, 1970 to 1997. Suicide Life Threat Behav 2002; 32: 191-208.

19 Worrall A, O'Herlihy A, Banerjee S, Jaffa T, Lelliott P, Hill P, et al Inappropriate admission of young people with mental disorder to adult psychiatric wards and paediatric wards: cross sectional study of six months' activity. BMJ 2004; 328: 867-8.

20 Parker $\mathrm{KCH}$, Roberts N, Williams C, Benjamin M, Cripps L, Woogh C. Urgent adolescent psychiatric consultation: from accident and emergency department to inpatient adolescent psychiatry. J Adolesc 2003; 26: 283-93.

21 Eastwood MR, Stiasny S. Psychiatric disorder, hospital admission, and season. Arch Gen Psychiatry 1978; 35: 769-71.

22 Hawton K, Hall S, Simkin S, Bale L, Bond A, Codd S, et al. Deliberate selfharm in adolescents: a study of characteristics and trends in Oxford, 1990-2000. J Child Psychol Psychiatry 2003; 44: 1191-8.

\title{
Assessing the security needs of patients in medium secure psychiatric care in Northern Ireland
}

\author{
Rowan James McClean ${ }^{1}$
}

The Psychiatrist (2010), 34, 432-436, doi: 10.1192/pb.bp.109.027672

${ }^{1}$ Knockbracken Healthcare Park, Belfast

Correspondence to Rowan James McClean

(rowanmcclean@doctors.org.uk)
Aims and method To determine the security needs of patients in medium secure services in Northern Ireland and to identify those requiring long-term secure care. Patients were rated on the Security Needs Assessment Profile.

Results Fifteen patients (45\%) could be managed in conditions of low security. Twelve patients (36\%) require over 5 years' further treatment in secure care. Particular security needs include relational nursing skills, restricted access to alcohol and drugs, and robust physical security.

Clinical implications This study should be replicated across the UK to determine whether forensic services are responding to patients' needs. Pathways out of medium security need to be better developed, with regional expansion of low secure services.

Declaration of interest None.
A lack of clear definitions of levels of security within psychiatric services has led to significant variation in purpose and characteristics of low and medium secure units throughout the UK. This problem is compounded by a paucity of reliable, valid tools suitable to assess an individual's security needs and match these to an appropriate unit. This study aims to assess the needs of patients within a new and developing medium secure unit in order to ensure that patients are appropriately placed and to guide future development of forensic services.

Medium secure units emerged throughout the UK in the 1980s following the Glancy and Butler reports. ${ }^{1-3}$ These units generally manage patients discharged from special hospitals (hospitals which treat psychiatric patients in high security because of their potential risk to others) or those transferred from prisons and courts. There is a wide variation between medium secure units across the UK, for example in the level of physical security provided. There is a similar variation in purpose and characteristics of the next step down in security, the low secure unit, which generally provides longer-term rehabilitation for chronically disturbed patients. As a result of this variation in standards and the large numbers of delayed transfers, published needs assessments have illustrated a considerable degree of inappropriate placement within the overall system. ${ }^{4}$ For example, Shaw et $a l^{5}$ found that $79 \%$ of patients in secure care in a sampled UK health region were placed at a level of security that was inappropriate to their needs.

Other problems have been noted with the current provision of low and medium secure units across the UK. Turner \& Salter ${ }^{6}$ highlight that beds in medium secure units are logjammed and that disputes over responsibilities and the appropriate use of resources have led to strained relations between forensic and general adult psychiatrists. 